\title{
Control of a train of high purity distillation columns for efficient production of $13 \mathrm{C}$ isotopes
}

\author{
Clara Ionescu, Roxana Both, Csaba Fustos, Clement Festila, Mihai Gligan and Robin De Keyser
}

\begin{abstract}
It is well-known that high-purity distillation columns are difficult to control due to their ill-conditioned and strongly nonlinear behaviour. The fact that these processes are operated over a wide range of feed compositions and flow rates makes the control design even more challenging. This paper proposes the most suitable control strategies applicable to a series of cascaded distillation column processes. The conditions for control and input-output relations are discusssed in view of the global control strategy. The increase in complexity with increased number of series cascaded distillation column processes is tackled. Uncertainty in the model parameters is discussed with respect to the dynamics of the global train distillation process. The main outcome of this work is insight into the possible control methodologies for this particular class of distillation processes.
\end{abstract}

Index Terms-multivariable control, uncertainty, process industry, distillation column, isotopes, carbon.

\section{INTRODUCTION}

$\mathbf{P}$ ROCESS control did not reach where it stands today if it wasn't for the continuous and strenuous efforts of control engineers for better and more efficient methods of tackling nonlinear, time-varying, multivariable and constraint problems [1]. The extensive literature survey from [2] on distillation dynamics and control suggests to the reader that the process of distillation is the most common unit of operation in the chemical industry and it represents a teacher's pet when it comes to illustrate control problems for training control engineers. Along with the numerous possibilities in tackling control issues, the process of distillation brings numerous constraints and challenges, being in itself a complete standalone process within the chain of industrial applications [1].

An excellent reader's digest on dynamics and control of distillation columns are [4] and [3]. Both of these vast works are a necessary guide through the jungle of various distillation column configurations and specifications for control. However, a special class of distillation processes are those aiming at stripping various chemical elements. There is a lack of papers in the literature discussing this particular class, despite renewed interest from the control community [5]. Most of the existing works on control of distillation columns examine the problem from an individual column standpoint. However, multi-column systems have been examined in cases where

C.M. Ionescu, R. Both, Cs.Fustos and C. Festila are with the Technical University of Cluj Napoca, Department of Control Engineering, 28 Memorandumului street, 400114 Cluj-Napoca, Romania.

R. De Keyser and C. M. Ionescu are with Ghent University, Department of Electrical energy, Systems and Automation, Technologiepark 913, B9052 Gent-Zwijnaarde, Belgium. Corresponding author: Clara M. Ionescu; tel: 0032-9-264-5608; fax: 0032-9-2645839.

M. Gligan is with the National Institute for Research and Development of Isotopic and Molecular Technologies (NIRDIMT), Cluj-Napoca, Romania. recycle streams are present between the columns creating chain interactions, with some applications in azeotropic distillation systems. Multiple columns in a plantwide environment have been explored in a variety of case studies of multiunit processes with reaction and separation sections. In these cases, multivariable model based (predictive) control is an excellent candidate for plantwide control with ability to tackle dead-times and constraints, either in global control, either in distributed control structures [6], [7].

The Carbon element has two stable isotopes: base component (12C) with the natural abundance of 98.89 at\% and the heavier stable isotope (13C), with 1.11 at\% concentration [9][11]. Using dedicated equipment, it is possible to detect if a substance contains a larger concentration of (13C), suggesting its synthetic origin. Based on this feature, the (13C) isotope may be used in different applications and fields like scientific research, bio-medicine, environmental protection, etc [8]. The carbon monoxide with high-purity in (12C) is used in electronics technology to produce synthetic diamond crystals with $50 \%$ better thermal conductivity than that of the usual synthetic crystals [9]. It is also very effective in detecting tumour in the human body [18] and broadly used in other clinical investigations [17]. An effective method to produce substances with high-concentration of these valuable isotopes is based on the cryogenic distillation of carbon-monoxide [9].

There are very few plants worldwide which can perform this complex operation. Among them is the National Institute for Research and Development of Isotopic and Molecular Technologies (NIRDIMT) from Cluj-Napoca, Romania [16]. The current bottleneck in this problem is the low efficiency of the high-purity stripping distillation, making the process unattractive from an economical point of view. The very complex stripping process requires large scale setups, without implicit efficiency. A pragmatic approach to solve this issue is to use trains of striping distillation columns. However, when closed loop control is involved, series of distillation columns with recycle streams can endanger the stability of the overall process and care must be taken when choosing the control configuration. The objective of this paper is to discuss these problems and propose solutions for safe operation and efficient (13C) isotope production.

The structure of the paper is as follows: first, some theoretical background on controlling distillation columnsis presented, followed by a numerical example of the column for stripping the $(13 \mathrm{C})$ isotope. 


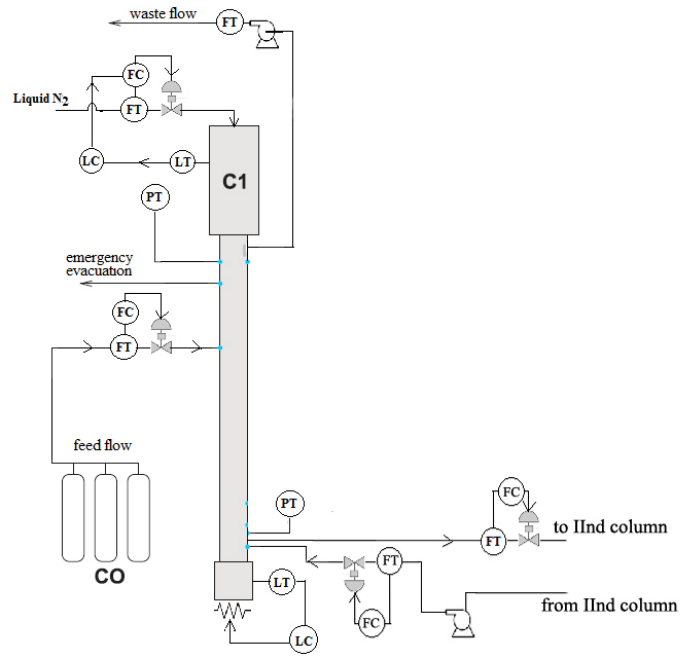

Fig. 1. Schematic of one CO stripping distillation column with possibility to series connect to a second column.

\section{THE ISOTOPE SEPARATION COLUMN}

There are several available methods able to increase the concentration of the (13C) isotope in some substances. One of them is that of cryogenic distillation of carbon monoxide [9]. For pure carbon-monoxide situations, at the vaporization temperature of nitrogen $\left(\approx 190^{\circ} \mathrm{C}\right)$, the liquid and gaseous phase co-exist [16]. Because the maximum vapor pressure by $(12 \mathrm{CO})$ is higher than the pressure of $(13 \mathrm{CO})$, the $(13 \mathrm{C})$ accumulates in the liquid phase, where it may be collected and extracted as final product. In the static contact of both phases, the increase in concentration is very small. As given by the separation factor $(\alpha)$, here we have a value very close to unity, i.e. $\alpha \simeq 1.011$ [9]-[11]. A continuous counter current of an ascendant gaseous stream and a descendent liquid stream of carbon-monoxide increases the (13C) concentration in the liquid phase and decreases it in the gas phase, which is then evacuated as waste. One dedicated equipment can offer an enrichment in (13C) only up to $8-10$ at\%, due to the above described physical limitations.

The (13C) isotope separation column of the National Institute for Research and Development of Isotopic and Molecular Technologies (NIRDIMT) from Cluj-Napoca is a steel packed pipe as depicted in Fig. 1, fed with pure gaseous carbonmonoxide by the flow-rate and (13C) isotope concentration at $1.11 \%$ at [9]. The isotope of interest (13C), accumulated in the liquid carbon-monoxide, is withdrawn as final product at the flow rate and concentration. The gaseous carbon monoxide with lower (13C) concentration is evacuated as waste at the top side, by flow rate and the concentration, which is in fact the secondary product of the separation column. The efficient thermal isolation of the column is based on the external vacuum jacket. If cryogenic distillation temperature is used, the vapor pressure of carbon monoxide with (12C) is greater than the pressure of carbon monoxide with (13C), hence the isotope (13C) accumulates in the liquid phase. This process is called the rectifying process. Simultaneously, a decrease in the concentration of (13C) in gaseous phase, is achieved during the stripping proces. In order to rise the (13C) isotope concentration up to a desired level, a permanent countercurrent of the liquid-gaseous phases must be ensured. This is provided by an electrically heated boiler in the column base and a condenser, cooled with liquid nitrogen at the column top side. The liquid nitrogen level in the condenser and the liquid carbon-monoxide in the boiler must be maintained at a constant level for optimal operation of the column. The generic task of the control system is to keep the (13C) isotope transfer rate constant, which can be achieved by various control configurations.

\section{Single-Column Control Configurations}

\section{A. Classical Control Approach}

Each distillation column is unique through its design and structure and a generic rigorous modelling and control approach is not a realistic objective. However, to some extent, distillation columns for separation processes have some common properties, such as thermodynamic principles and basic dynamics, which will be employed hereafter to discuss the classical control approach.

Typically, a high purity distillation column is connected in series to another train of distillation columns [5]. The bottom product streams of these prior columns are buffered into a level-controlled tank and fed into the high-purity column (HPC). Hence, the feedflow is varied stepwise instead of continuous. However, the variations in the composition are smooth. In case of a stripping process of series of HPCs, the top product streams directly into the feedflow of the next HPC. In this case, smooth continuous variations in the feedflow are expected as well as feed compositions. The monitored variables are typically top pressure in the column and top level control of the cooling liquid.

The composition dynamics are most important for control since variations in one HPC in series will affect the input, hence the dynamics of the next HPC. The product compositions are a nonlinear function of the reflux, boilup, and feed comdition. For instance, a 5\% increase of the reflux flow rate improves the top product composition with a certain amount, but the same percent decrease degrades it much more. There are also very strong interactions in the HPC; e.g. a change of reflux or boilup alters both product compositions. The interaction between both product compositions and reflux and boilup has a severe consequence for the composition dynamics, known as ill-conditioned behaviour.

The control objectives of HPC can be summarized as threefold:

- control of the material balance

- product quality control

- maintaining constraints

The first objective includes the control of the vapor holdup (top pressure), the reflux accumulator level, and the reboiler level. Practically, these control objectives are easily achieved by simple PI controllers. The second objective is the most important since it is related to the optimal economic and ecologic operation of the DHC [21]. It is well known that 
tight control of both product qualities minimizes the energy comsumption and delivers within-specs products. However, this is a challenging control task due to the presence of disturbances such as variations of the feedflow rate and feed composition (recall that is a series train of HPC). Tight composition control requires advanced control schemes and has been the subject of several important works [2], [3], [19], [20]. Reflux, boilup and pressure drop are allowed to vary within a predefined range. Any operation of a distillation column outside this range may cause insufficient separation or even physical damage of the setup.

A typical control problem of HPC has five controlled variables: top composition, bottom composition, reflux accumulator level, reboiler level and top pressure; and has five manipulated variables: reflux, boilup (e.g. via reboiler duty), top product flow rate, bottom product flow rate, cooling liquid flow rate. Due to the high inherent sensitivity of multivariable controllers to sensor or actuator failure, the inventory control (product quality) and the composition control are independently designed, increasing robustness of the overall process.

In a first step, the two manipulated variables are selected for the composition control. In the specialized terminology, the choice of these manipulated variables names the control configuration. An excellent overview of the various possible configurations is given in [19], [20]. For instance, if the top composition is controlled by reflux (L) and bottom composition is controlled by boilup (V), the control scheme is denoted as LV. The remaining manipulated variables are thus available for level and pressure control.

If a model of the HPC exists, typically in the form of a linearized model around the optimal operating point, then the choice of the input-output variables can be done on methods as the relative gain array, Niederlinski index or singular value decomposition [4], [24]. In practice, the application of these methods may lead to considerably different solutions and dynamic effects due to the interaction of inventory and composition control are neglected.

The most common control configuration in the chemical industry is the LV configuration [20]. This control structure is rather independent of the inventory control dynamics and has been proven to have good experiemtnal results. In general, tight inventory control can be achieved with three simple PI controllers. Some distillation columns show an inverse response of the reboiler level to an increase of boilup. In this case, tight control with boilup as manipulated variable becomes challenging and the re-selection of the manipulated variable may be necessary. Inverse dynamics occur in the presence of a RHP transmission zero in the linearized model analysis; by changing the variable selection, such RHP zero may be avoided.

\section{B. Classical Modelling Approach}

Robust controllers are based on the existence of a (linear) process model [13]. The development of a good model is therefore crucial for the control system synthesis. These models should describe the dynamic behaviour of the process within a wide frequency range and they can be twofold:
- system identification models

- linearized models from nonlinear model

Obviously, system identification around the optimal (calculated) operating point is the most pragmatic approach, but it poses some difficulties:

- if time constants are large (depending on the construction design of the HPC), then the input-output records can be quite time-consuming;

- since the HPC has a high sensitivity to changes in the internal flow rates, it is difficult to define 'small' amplitude variations in the input which will not exceed the linear region;

- each identification experiment will cause disturbances in the product quality

- such models are valid only around the operating point where they were identified.

These dis-advantages make system identification not an interesting approach; instead, linearization of nonlinear models is preferred and modelling uncertainty is allowed to be tackled by the choice of the control structure. The problems arising from the linearization process are related to idealizing assumptions, such as:

- constant pressure drop

- constant and equal enthalpies on all trays

- constant total holdup on all trays (equimolar flow)

which of course, are not realistic. The first assumption means a neglect of the correlation between tray pressures, holdups, and boilup rates. The second assumption implies uniform vapor flows within the stripping section and within the rectifying section of the HPC. The assumption of a constant tray holdup neglects in part flow dynamics. These assumptions introduce in the model high frequency errors, which may be critical if a fast performance given by a large bandwidth of the closed loop is envisaged.

\section{Modelling Uncertainty}

Model uncertainty and lack of validity in the operating range can seriously affect the overall plant stability and robustness [14], [15]. Typical sources of model uncertainty for HPC are measurement errors, limited actuator speed, unmodelled high frequency dynamics, process nonlinearity. In the case of HPC, which are highly nonlinear plants, the error of a linear model rapidly increases with the distance from the operating point where it was identified/linearized. Additional stochastic disturbances will also affect the process dynamics, in which case the error cannot be determined. This error between the process model and the process itself can be modelled either as a single frequency-dependent uncertainty bound (i.e. unstructured uncertainty), or as several frequencydependent uncertainy bounds (i.e. structured uncertainties).

Several types of uncertainty bounds can be implemented, tackling various practical aspects. Of these, we mention:

- input uncertainty

- model uncertainty

- measurement uncertainty

and we discuss the essential solutions for each of them hereafter. 


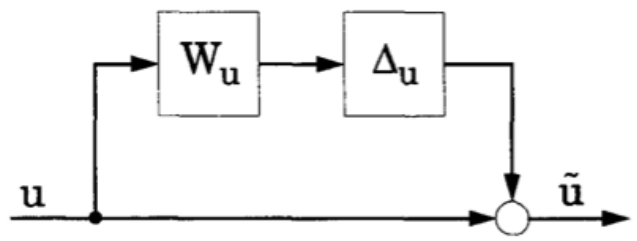

Fig. 2. Multiplicative uncertainty for the HPC input.

The actual values of the manipulated variables reflux and boilup will never match exactly the values requested by the control system. Moreover, this error will also be frequency dependent, because: i) static and dynamic measurement errors of reflux and reboiler duty; ii) actuator resolution; iii) changing heat of evaporation due to pressure and temperature variations; iv) reboiler and actuator lags; v) effects of sampling (i.e. digital systems).

The bounds for relative errors in the column inputs $u$ can be modelled by a multiplicative uncertainty description with the frequency-dependent error bound $w_{u_{L}}$ for the reflux $\mathrm{L}$ and the error bound $w_{u_{V}}$ for the boilup V. These bounds are combined in the matrix $W_{u}$ as in figure 2 .

The input uncertainty model is given by:

$$
\tilde{u}(j \omega)=\left\{I+\Delta_{u}(j \omega) W_{u}(j \omega)\right\} u(j \omega)
$$

with

$$
\left\|\Delta_{u}(j \omega)\right\|_{\infty} \leq 1
$$

and

$$
W_{u}(j \omega)=\left[\begin{array}{cc}
w_{u_{L}}(j \omega) & 0 \\
0 & w_{u_{V}}(j \omega)
\end{array}\right]
$$

It has been shown in [22] that the performance of the closed loop for a HPC is very sensitive to errors in the manipulated variables. For controller design (and analysis) the error bounds $W_{u}$ need to be estimated as good as possible, especially in the low frequency range where the condition number of the column model is high. The risk is lower control performance if overestimation occurs. In this low frequency range, the errors of the manipulated variables at the plant input are strongly dominated by flow measurement errors and parameter variations. For instance, if boilup is controlled indirectly by the steam flow rate, a change in the heat of evaporation will cause errors in vapor flow rate leaving the reboiler. Calibration of the flow meters is therefore crucial.

The effects of reboiler lags, actuator lags, dynamic measurement errors and sampling period are present in the high frequency range. These errors increase with the frequency and can easily exceed $100 \%$ of the nominal values for frequencies above $1 \mathrm{rad} / \mathrm{min}$. In this case, the steady-state error together with the high-frequency error can be approximated by a first order lead/lag transfer function:

$$
G(s)=K \frac{1+s / \omega_{N}}{1+s / \omega_{D}}
$$

with $\omega_{N}, \omega_{D}$, the gain $K$ represents the steady state error and cut-off frequencies chosen for $\omega_{D}>10 \omega_{N}$.
The model uncertainty has twofold origins: i) column nonlinearity and ii) un-modelled dynamics. The highly nonlinear behaviour of HPC is observed at varying operating points and at transients during disturbance rejection. This depends on the varying internal flow rates ( $\mathrm{L}$ and $\mathrm{V}$ ) and on the composition profile within the distillation column, represented by the liquid and vapor phase compositions.

Any type of control system for HPC will exhibit large gains in the low frequency range to achieve small control errors at steady-state. Transients have no significant effect in the low frequency range and the internal vapor and liquid flow rates as well as the composition profile within a column become a function of feed flow rate and composition only. However, the dynamic behaviour of a HPC depends substantially on the true composition profile and the true internal vapor and liquid flow rates. Consequently, the operating range of a HPC can be bounded with a maximum and a minimum feed flow rate and composition. In this framework, it follows that the largest internal flow rates will exist for the smallest feed composition and largest feed flow rate. Similar reasoning indicates that the smallest internal flow rates will exist for the largest feed composition and smallest feed flow rates. hence, the low frequency behaviour of a HPC is bounded by the models for maximum and minimum column load.

The simplest way to represent the column nonlinearity due to varying operating points would be to use a multiplicative output uncertainty. Assuming the uncertainty for each model output independent of the actual value of the other model outputs, one has:

$$
\tilde{y}(j \omega)=\left\{I+\Delta_{y}(j \omega) W_{y}(j \omega)\right\} y(j \omega)
$$

with

$$
\left\|\Delta_{y}(j \omega)\right\|_{\infty} \leq 1
$$

and

$$
y(j \omega)=G_{N}(j \omega)\left[\begin{array}{l}
d(j \omega) \\
u(j \omega)
\end{array}\right]
$$

with $G_{N}$ the transfer function matrix of the plant at nominal operating point and $W_{y}$ a diagonal matrix similar to (3). Care must be taken with such uncertainty representation since the low frequency range tends to have a high multiplicative gain, which is prohibited for any control design. However, things become simpler if the errors are highly correlated, i.e. the variation of the steady-state operating points causes a simultaneous increase/decrease of the singular values of the transfer functions from the control signals $u$ to the model outputs $y$. This statement can be evaluated by means of Nyquist plots for individual channels $u_{i} \rightarrow y_{i}$. Notice that the effect of transients obeys the laws of a rather unstructured uncertainty. Due to the nonlinear vapor/liquid equilibrium, the singular values of the transfer functions $G_{u \rightarrow y_{j}}$ may change in different directions, which pose other control challenges.

The second origin for model uncertainty was un-modelled dynamics. Most of the works in literature treat the effect of flow dynamics in an input time delay $\tau$, with $0<\tau<1$ minute [23]. 
Finally, measurement uncertainty can be well exemplified by temperature measurements. These can be well approximated by a first order lag modelling the behaviour of a temperature sensor. The time constant of this model depends on the position of the temperature sensor. If the sensor is placed in the liquid phase, time constant of the order of 1 minute can be expected. However, if the sensor is placed in the vapor phase, it can go up to 10 minutes or more. The gain of the model is related to sensor calibration and heat loss to the environment. For the case of our HPC, heat losses are close to zero (vacuum jacket) and calibration is assumed to be correct.

The complete uncertainty model consists of input uncertainty (1), model uncertainty (5) and performance specifications. Simple dynamic models are used whenever possible, to avoid further uncertainy blocks. The advantage of such modelling approach is that the entire operating range of the HPC is covered.

\section{Improved Control Approach}

From an economic point of view, disregarding the control and measurement problems, two-point control is obviously the best, i.e. both composition loops closed. This follows since the optimal operating point corresponds to some given purity specification. In case of stripping HPCs, these are not independent. There is also a case when one-point control is optimal, i.e. when the column is operated at maximum capacity. In the LV configuration, by controlling one composition, inherently we control the other (i.e. strongly coupled). If one can measure the feed rate, and implement a feedforward controller by using the ratio $\mathrm{L} / \mathrm{F}$, then we achieve self-regulation of $\mathrm{F}$. In this way, one achieves acceptable control of both products (top and bottom) if single end control of bottom composition is used and fixed L/F ratio.

Feed composition sensitivity analysis is used to determine of a single-end control structure would be effective. This is achieved with dedicated software, based on steady-state simulations. Typically, one checks the percent variation of the required reflux flow rate and reflux ratio over the range of feed compositions. Depending on the estimated values, it may be indicated that for HPC a single end control structure with a fixed reflux-to-feed ratio would outperform a fixed reflux ratio structure [5]. The basic control structure is depicted in figure 3 and suggested improved structure in figure 4 as following. In a train of series HPC, the feed comes in on flow/level control from the upstream column. Pressure is controlled by manipulating condenser duty. Reflux can be ratioed to feed, and reflux-drum level is controlled by manipulating the flow rate of the distilate product. Base level is controlled by manipulating the flow rate of the bottoms. Temperature is not controlled directly, but from the pressure measurements at top and bottom HPC trays, its reference value can be estimated and controlled by reboiler duty.

Competing rules for distillation control need to be negociated. For instance, analysis suggests that the reflux-drum level is controlled by reflux because of very high reflux ratio. However, it also suggests that the reflux should be ratioed

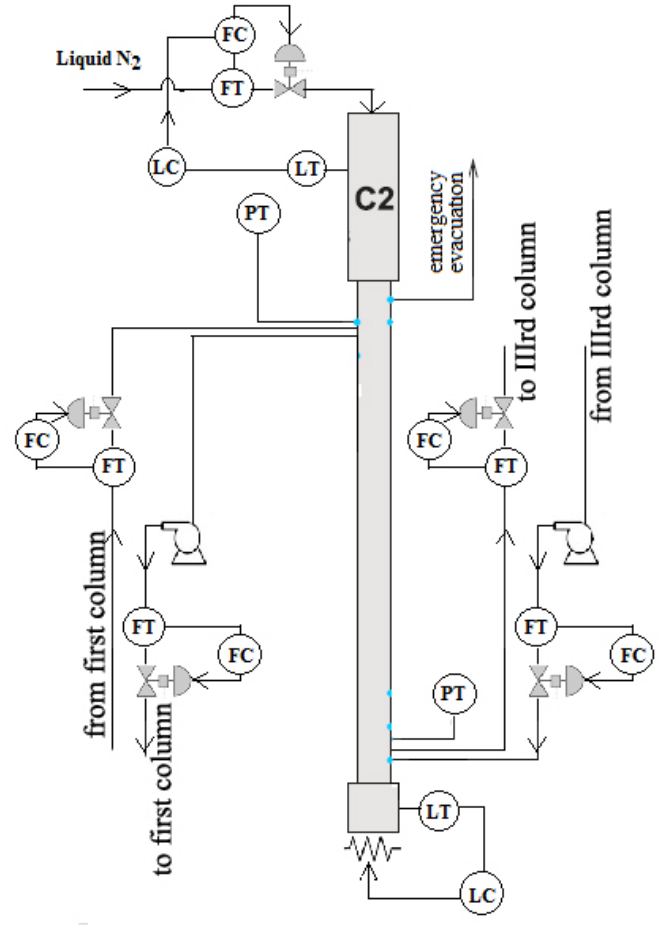

Fig. 3. Schematic of a basic control structure of one HPC within a train of series HPCs.

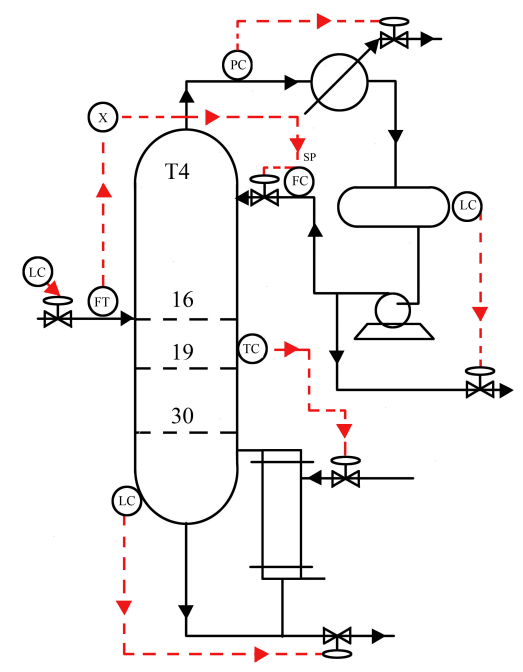

Fig. 4. Proposed control structure of one HPC within a train of series HPCs.

to the feed. Obviously, these are not independent, hence cannot be achieved simultaneously. An ingenious solution is given in [5], summarized as follows and illustrated in figure 5. This control structure is similar to the previous one 


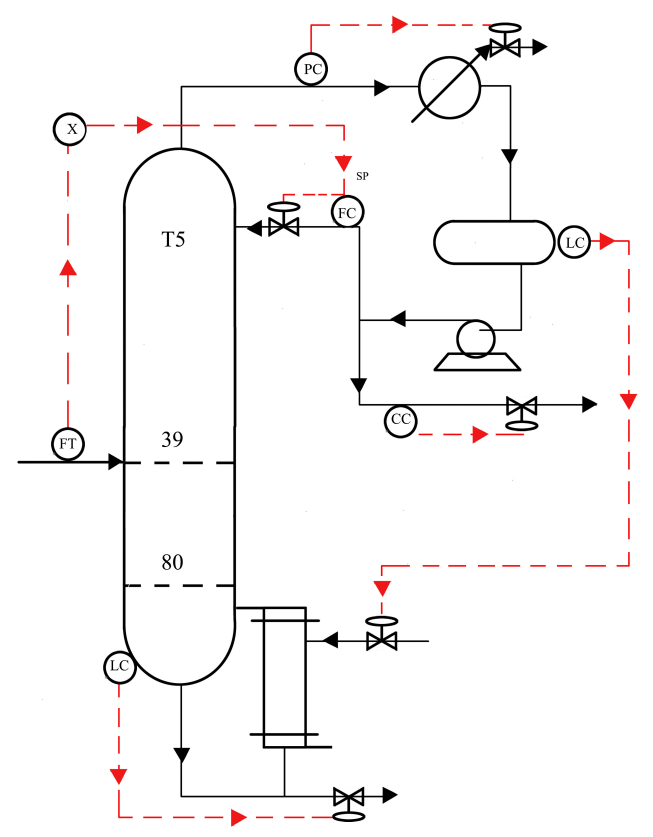

Fig. 5. Another proposed control structure of one HPC within a train of series HPCs.

proposed, except the following: reflux-drum level is controlled by manipulating reboiler duty and the distillate impurity is controlled by manipulating the control valve in the distillate line.

The control structure from figure 5 is unusual because of the use of reboiler heat input to control the reflux-drum level. This is based on the assumption that the feed composition sensitivity analysis requires very small changes in the ratio, hence it can be fixed. The liquid level in the reflux drum can be controlled by distillate - this will fail if the flow rate is much smaller than the reflux. In the latter case, the reboiler duty can be employed efficiently to solve this dilemma, if the changes in vapor flow rates in the column are fast. The distillate composition is controlled by the distillate flow rate. Using high gain control, it is possible to achieve faster response of vapor boilup to level changes. These are then detected by the level controller, which changes the vapor rate up the column and affects distillate composition. Notice that the pressure controller manipulating condenser duty is nested inside the composition control loop.

Two-point control will not be adressed here, but we may suggest some ideas. The liquid flow dynamics decouple the two column ends (top and bottom) at high frequency. If effort is put into making the quality loops fast enough, good control can be achieved for both compositions. This can be done using temperature measurements, or in our case inference from pressure measurements, with an outer composition cascade loop. In other words, a combination of the control schemes suggested in figure 4 and figure 5.

\section{NUMERICAL EXAMPLE}

\section{A. Single column}

For the column from Fig 1, the transfer function matrix is given by:

$$
\left[\begin{array}{ccc}
\frac{-0.111}{s^{2}+1.094 s+0.0842} & \frac{0.1152}{s^{2}+1.211 s+0.2021} & 0 \\
\frac{-0.0017}{s^{2}+0.1343 s+0.0019} & \frac{0.0038}{s^{2}+0.1547 s+0.0043} & \frac{-1.104}{s+0.1176} \\
\frac{-0.0099}{s^{2}+1.056 s+0.0703} & \frac{0.0062}{s^{2}+1.085 s+0.0985} & \frac{8.457}{s+0.9851}
\end{array}\right]
$$

where the time delay has been ignored for simplicity and with inputs and outputs verified by the relative gain array as following: i) first manipulated input is the output waste flow from the column to control the pressure in the column at the condenser zone, ii) the second manipulated input is the feed flow to the column to control the liquid carbon monoxide level in the boiler, and iii) the third manipulated input is the electrical power supplied to the boiler resistor, to control the pressure in the column at the boiler zone. The dependence of the relative gain array with frequency depicted in Fig 6 suggests that for step changes in the controlled variables, the pairing is optimal.

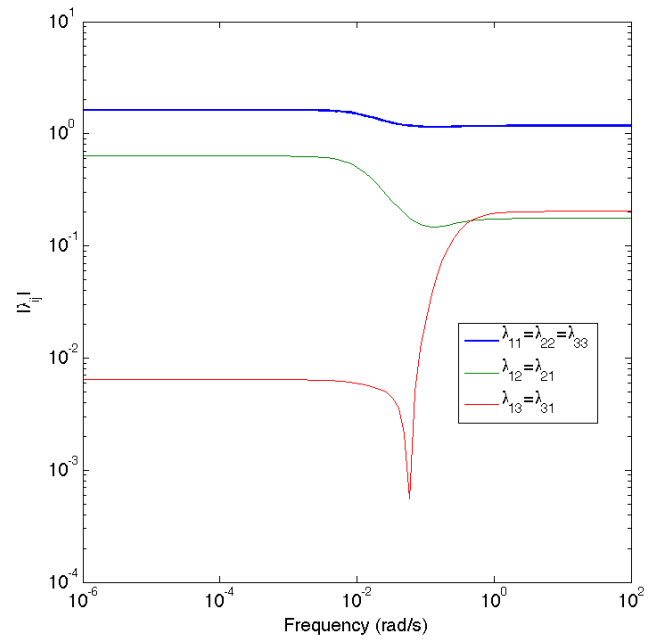

Fig. 6. Dependance of frequency of the relative gain array for one column.

Further analysis into the magnitudes of the transfer function matrix from (8) suggests that the bandwidth of the system is around $0.1 \mathrm{rad} / \mathrm{s}$, as in Fig 7.

Finally, the singular value decomposition suggests that the first pair of input-output variables may pose most difficulty for control if the bandwidth of the system is increased to obtain better perfromance in terms of smaller settling times. Fig. 8 depicts these values as a function of frequency.

\section{B. The train of distillation columns}

The real life setup deals with a cascade of three columns [16]. The permanent counter-current of liquid and gaseous phase is ensured by a common condenser cooled with liquid nitrogen and by three different boilers, one for each column, heated with electrical resistors. Studying the coupling possibilities of the three columns, the conclusion was to feed 


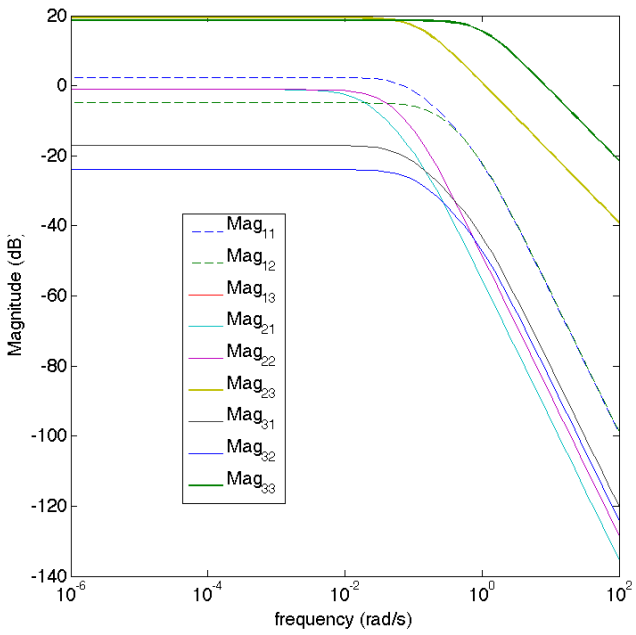

Fig. 7. Gains of the system for one column.

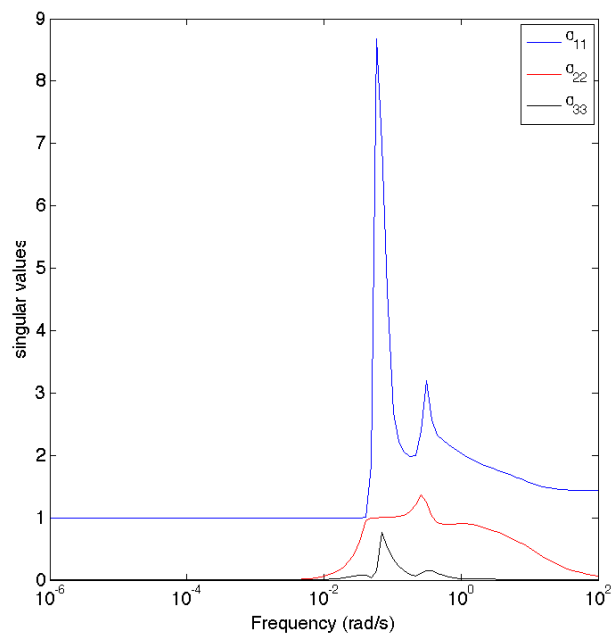

Fig. 8. Singular values of the system for one column.

the first column with carbon monoxide, the enriched gaseous phase from the first column being used as feed for the second column and the product of the second column as feed for the third column, ensuring maximum enrichment in (13C) with minimum equipments. Previous studies revealed that the major influence on the end product in a separation column has the vapor upstream, which is maintained by the electrical resistor of the boiler. This was the reason why the authors have chosen to implement in present work the distributed control of the resistor powers for the cascade of three columns.

For the second column from Fig 9, the transfer function matrix is given by:

$$
\left[\begin{array}{ccc}
\frac{-0.111}{s^{2}+1.111 s+0.1011} & \frac{0.1152}{s^{2}+1.311 s+0.3033} & 0 \\
\frac{-0.0017}{s^{2}+0.1300 s+0.0022} & \frac{0.0038}{s^{2}+0.1500 s+0.0044} & \frac{-1.104}{s+0.1200} \\
\frac{-0.0099}{s^{2}+1.060 s+0.0784} & \frac{0.0062}{s^{2}+1.105 s+0.1225} & \frac{8.457}{s+0.9800}
\end{array}\right]
$$

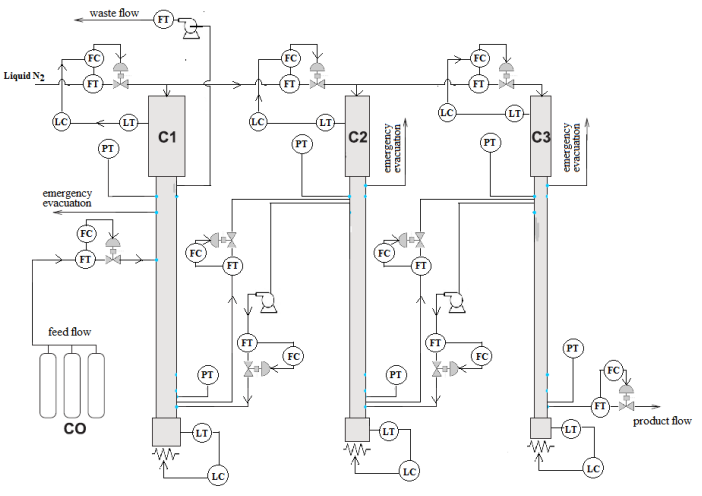

Fig. 9. Schematic of the stripping train of distillation columns.

and for the third column is given by:

$$
\left[\begin{array}{ccc}
\frac{-0.111}{s^{2}+1.131 s+0.1213} & \frac{0.1152}{s^{2}+1.361 s+0.3538} & 0 \\
\frac{-0.0017}{s^{2}+0.145 s+0.003} & \frac{0.0038}{s^{2}+0.1700 s+0.0060} & \frac{-1.104}{s+0.1400} \\
\frac{-0.0099}{s^{2}+1.085 s+0.0985} & \frac{0.0062}{s^{2}+1.120 s+0.133} & \frac{8.457}{s+0.9850}
\end{array}\right]
$$

The interaction matrices for the reflux from the second column to the first and from the third column to the second are given by

$$
\left[\begin{array}{ccc}
0 & 0 & 0 \\
\frac{1}{10 s+1} & \frac{1}{10 s+1} & 0 \\
0 & 0 & 0
\end{array}\right]
$$

and

$$
\left[\begin{array}{ccc}
0 & 0 & 0 \\
\frac{1}{7.5 s+1} & \frac{1}{7.5 s+1} & \frac{1}{7.5 s+1} \\
0 & 0 & 0
\end{array}\right]
$$

, respectively.

From a practical standpoint, all loops within a HPC can be taken as P-controlled (proportional gain only). In this way, fast dynamics can be achieved. However, since composition feedback control usually has slow dynamics, a PI-control can be used as an outer loop. This results in a cascaded control scheme, which seem to be the optimal solution from the afore mentioned analysis. The controller parameters are given by:

$$
\begin{gathered}
C 11(s)=-\frac{0.4923 s+0.03678}{s} \\
C 22(s)=\frac{1.937 s+0.03622}{s} \\
C 33(s)=\frac{0.16 s+0.16}{s} \\
C 44(s)=-\frac{0.3881 s+0.04709}{s} \\
C 55(s)=\frac{1.835 s+0.04126}{s} \\
C 66(s)=\frac{0.17 s+0.17}{s} \\
C 77(s)=-\frac{0.3156 s+0.05707}{s} \\
C 88(s)=\frac{1.899 s+0.07255}{s}
\end{gathered}
$$




$$
C 99(s)=\frac{0.18 s+0.18}{s}
$$

Analysis of the sensitivity functions reveal in Fig. 10 that the most difficult to control is the first column, since all dynamics propagate from it into the output of the last column, as per global control objective.

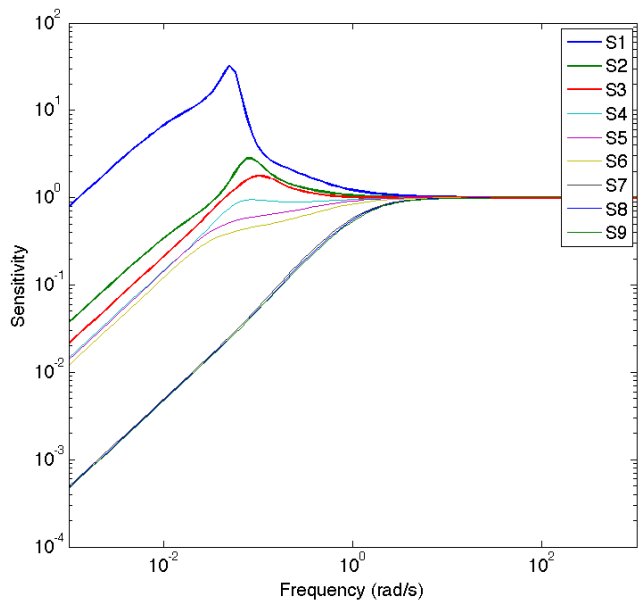

Fig. 10. Schematic of the stripping train of distillation columns.

The most economic control scheme will only use one distillate composition sensor at the output of the third HPC. In this case, there are several possibilities for global control. First possibility is to use cascade control with composition of the third column output as the outer loop and temperature (via pressure) control in the same column. The first and second HPC are not controlled for composition output, only kept within a desired (i.e. calculated) range for level, feed flow rate and pressure values. This is a relatively simple solution, if tight control is not necessary. Second possibility is to use tight control in each HPC, and global control for composition, measured at the output of the third column. This implies a hyerarchical or distributed control strategy, where each column is controlled separately for its optimal operation, but since they interact with each other, a global cost index (typically related to the composition) provides the operating conditions and reference values for each of them. This is a more complex problem in terms of stability, but still numerically acceptable for real-life implementation. Third possibility is to view the ensemble of the series train of HPCs as a input-output block, with intermediate variables, in a centralized control strategy. Intuitively, inputs will be those entering the first column and outputs will be those from the third column. The drawbacks of this scheme are the model complexity and the intrinsic coupling effects between columns due to recirculation, as depicted in figure 9 . The latter can be avoided by very good condenser and reboiler operation, i.e. avoiding high variations in the pressures at the column ends.

\section{CONCLUSION}

This paper presented some first ideas on the control of a train of high purity concentration distillation columns. The study case was developed around the stripping process of the (13C) isotope, a valuable product in a manifold of applications. As the control methodology is further developed, insight into the problem will become available to further improve this complex process.

\section{ACKNOWLEDGMENT}

This work was supported by a grant of the Romanian National Authority for Scientific Research, CNDI UEFISCDI, project number 155/2012 PN-II-PT-PCCA-2011-3.2-0591.

\section{REFERENCES}

[1] B. W. Bequette, "Process Control: Modeling, Design and Simulation", Prentice Hall; 1 edition (2003)

[2] S. Skogestad, "Dynamics and control of distillation columns - a critical survey", Modeling, Identification and Control, 18, pp. 177-217, (1997)

[3] S. Skogestad, "Dynamics and control of distillation columns - A tutorial introduction", Trans IChemE, 75, Part A, pp. 539-562, (1997)

[4] W. L. Luyben, "Process Modeling, Simulation, And Control For Chemical Engineers", Published by McGraw-Hill (1973)

[5] W. L. Luyben, "Control of a train of distillation columns for the separation of natural gas liquid", Ind Eng Chem Res, 52, pp. 10741-10753, (2013)

[6] E.F. Camacho, C. R. Bordons, "Model Predictive Control", Springer, 2nd ed. (2004)

[7] J-N. Rico, "Control of dead-time processes", Springer, (2007)

[8] http://www.iso-analytical.co.uk/carbon.html

[9] D. Axente, M. Abrudean, A. Baldea, "Isotope Separation of 15N, 18O, 10B, 13C by isotopic exchange", (in Romanian), Casa Cartii de Stiinta, Cluj-Napoca, (1994)

[10] K. Cohen, "The Theory of isotope separation as applied to the largescale production of U235", McGraw-Hill Book Company, Inc, (1951)

[11] H. London, F.R.S. Phil, "Separation of Isotopes", George Newnes Limited, Tower House, London, (1962)

[12] C.I. Pop, C.M. Ionescu, R. De Keyser, "Time delay compensation for the secondary processes in a multivariable carbon isotope separation unit", Chem Eng Sc, 80, pp. 205-218, (2012)

[13] E.H. Dulf, C.I. Pop, C. F. Dulf, "Systematic Modeling of the (C-13) Isotope Cryogenic Distillation Process", Separation Sc and Tecn, 47(8), pp. 1234-1240, (2012)

[14] C.I. Pop, C. Festila, E.H. Dulf, "Optimal Control of the Carbon Isotopes Cryogenic Separation Process", Chem and Biochem Eng Quart, 24(3), pp. 301-307, (2010)

[15] , H. Li, Y. Ju, L. Li, "Separation of isotope C-13 using high-performance structured packing", Chem Eng and Proc, 49(3), pp. 255-261, (2010)

[16] A. Radoi, M. Gligan, S. Dronca, "Experimental plant for C-13 enrichment by carbon oxide distillation at low temperatures", Revista de Chimie, 50(3), pp. 221-225, (1999)

[17] A. Modak, "Stable isotope breath tests in clinical medicine: a review", J Breath Res, 1(1), Article Number: 014003, (2007)

[18] T. B. Rodriguez, E.M. Serrao, B.W. Kennedy, D. Hu, M. Kettunen, K.M. Brindle, "Magnetic resonance imaging of tumor glycolysis using hyperpolarized 13C-labeled glucose", Nature Medicine, 20(1), pp. 9397, (2013)

[19] S. Skogestad, M. Morari, "Control configuration selection for distillation control", AIChE J, 33(10), pp. 1620-1635, (1987)

[20] S. Skogestad, P. Lundstrom, E. Jacobsen, "Selecting the best distillation control configuration", AIChE J, 36(5), pp. 753-764, (1990)

[21] F. Shinskey, "Distillation control for productivity and energy conservation", 2nd ed. McGraw-Hill, New York, (1984)

[22] S. Skogestad, M. Morari, J.C. Doyle, ”Robust control of ill-conditioned plants: high purity distillation", IEEE Trans Autom Ctrl, 33(12), pp. 10921105, (1988)

[23] S. Skogestad, P. Lundstrom, "Mu-optimal LV control of Distillation Columns", Comp. Chem. Eng., 14(4/5), pp. 401-413, (1990)

[24] S. Skogestad, I. Postlethwaite, "Multivariable feedback control", John Wiley \& Sons, (2005) 